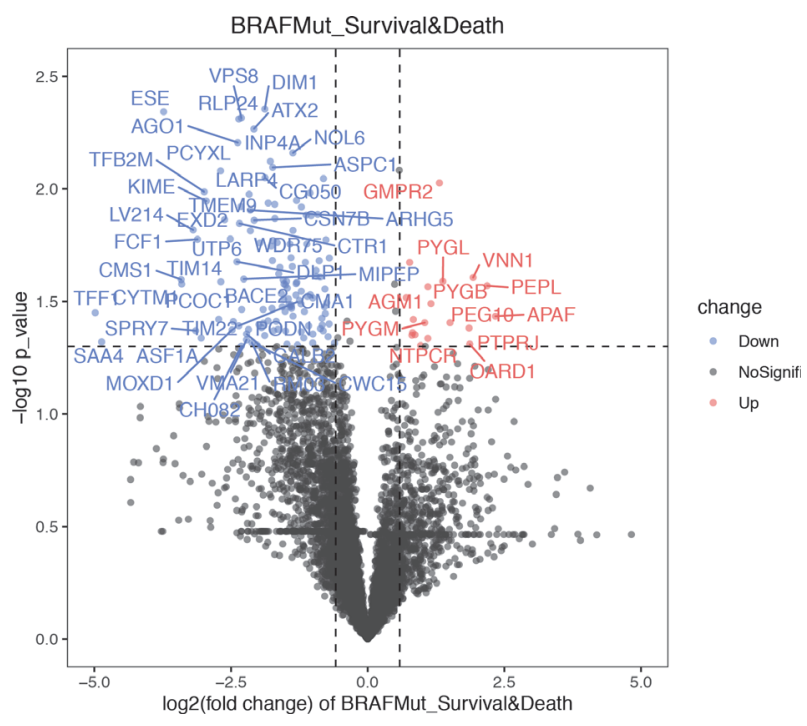

Abstract IDDF2020-ABS-0070 Figure 2

upregulated and 33 downregulated in BRAFV600E mutation versus matched wild type (figure 1). The most significant biological processes for overexpressed proteins are interferon-gamma-mediated signaling pathway and antigen processing and presentation of exogenous peptide antigen, while for downregulated proteins is ncRNA metabolic process. In BRAFV600E mutated samples, 151 differentially expressed proteins were obtained, including 131 downregulated and 20 upregulated from patients' overall survival less than 5 years versus exceeding 5 years (figure 2). The overrepresented biological process and molecular functions are leukocyte mediated immunity and SHG alpha-glucan phosphorylase activity for overexpressed proteins, while ribosome biogenesis and RNA binding for downregulated proteins.

Conclusions Based on the general and primary proteomic analysis, we found that BRAFV600E mutated CRC exhibited immune-related characteristics, and the poor prognosis might relate to the abnormal metabolic process. Relevant mechanisms and pathways will be studied in further research.

\section{IDDF2020-ABS-0072 EFFECT OF COMPREHENSIVE NURSING INTERVENTION ON PERIOPERATIVE PSYCHOLOGICAL AND STRESS STATUS OF ERCP}

Wen-hui Tan*, Xiao-qin Zhang, Dong-hong He, Fang Wang. Guangdong Second Provincial General Hospital, China

\subsection{6/gutjnl-2020-IDDF.60}

Background To investigate the effect of comprehensive nursing intervention on the psychological and stress status of ERCP patients during the perioperative period.

Methods A total of 100 patients who underwent ERCP in our Endoscopy Center from July 2018 to July 2019 were enrolled for the research. The first 50 patients were selected as a control group and given routine care in chronological order, while the last 50 patients were selected as observation group who were implemented perioperative comprehensive nursing intervention on a routine basis. A psychological evaluation was performed on both groups of patients after operation.

Results The anxiety score $(14.01 \pm 0.11)$ and depression score $(15.23 \pm 1.48)$ of the observation group were significantly lower than those of the control group $(\mathrm{P}<0.05)$; stress status of the observation group (slightly 30 cases (60.00\%), moderate 20 cases(40.00\%)) was significantly different from those of the control group $(\mathrm{P}<0.05)$; the complication rate in the observation group was $4.00 \%$ (1 case of acute pancreatitis, 1 case of cholangitis) and it was significantly lower than that in the control group $(\mathrm{P}<0.05)$.

Conclusions The implementation of comprehensive nursing intervention for ERCP patients during perioperative period can effectively improve the negative emotions of patients, improve the stress status of patients, reduce the incidence of complications, and be beneficial to postoperative recovery.

\section{IDDF2020-ABS-0074 ANALYSIS OF COAGULATION FUNCTION IN PATIENTS WITH INFLAMMATORY BOWEL DISEASE}

Yi Yu*. The Second Affiliated Hospital of Guangzhou University of Chinese Medicine, China

\subsection{6/gutjint-2020-IDDF.61}

Background To study the related factors of hypercoagulability in patients with inflammatory bowel disease (IBD) and their relationship with CRP, ESR and D-D, and to screen out the population with the highest risk of hypercoagulation, so as to prevent the formation of thrombosis in the early stage.

Methods We directly extract data from the MIMIC-III and Philips eICU collaboration databases. 739 cases were met; the diagnostic standard was included and divided into UC group and $\mathrm{CD}$ group. The patients were divided into groups of 0 20 years old, 20-40 years old, 40-60 years old and 60-80 years old, and divided into male and female according to gender. The patients with UC were divided into mild group, moderate group and severe group according to the improved Truelove and Witts severity. The patients hospitalized with polyps of digestive diseases were chosen as normal control group. Record data about blood routine examination, coagulation function and D-D were also collected.

Results There were statistical differences in TT and FIB between patients with IBD and those with polyps $(\mathrm{P}<0.05)$, but no statistical differences in APTT. There was no statistical difference between patients with UC and patients with $\mathrm{CD}$ in TT, FIB and APTT. There was no statistical difference in the analysis of TT and FIB among IBD patients in terms of age. There were no statistical differences in TT and FIB among UC patients in terms of gender and age; FIB was statistically different in the disease severity with the active phase, while TT was not statistically different.

\section{Conclusions}

1. IBD patients have higher coagulation status than normal people. The hypercoagulability of IBD patients was correlated with ESR, but not with CRP and D-D.

2. The higher the age of $\mathrm{CD}$ patients, the higher the risk of coagulation. The risk of hypercoagulability in UC patients was independent of gender and age; The more serious the condition was in the active stage, the more significant the hypercoagulability was.

3. The hypercoagulability of UC patients has little correlation with ESR and D-D, but no correlation with CRP. 